\author{
Maria Carmela APRILE, \\ Francesco BUSATO, \\ Francesco GIULI, \\ Enrico MARCHETTI
}

\title{
PROSPECT THEORY AND TAX COMPLIANCE: A MICROFOUNDED EQUILIBRIUM PERSPECTIVE
}

\begin{abstract}
This paper discusses the capabilities of a class of microfounded equilibrium models, augmented with Prospect Theory elements in the spirit of alNowaihi and Dhami (2007), to address several open questions in the analysis of tax evasion and compliance decisions. There are three main results: i) there ex-

(C) Maria Carmela Aprile, Francesco Busato, Francesco Giuli, Enrico Marchetti, 2019.

We are grateful to Giuseppe Ciccarone, John B. Donaldson, Paolo Siconolfi, for comments and suggestion. Of course all errors are ours.

Aprile Maria Carmela, Corresponding Author. University of Naples «Parthenope», Department of Economic and Legal Studies, Via G. Parisi 13, 80132 Naples, Italy. Tel. +39 081 5474339;Fax: +39 081 5474750. E-mail address: aprile@uniparthenope.it.

Busato Francesco, University of Naples «Parthenope», Department of Economic Studies, Via G. Parisi 13, 80132 Naples, Italy.E-mail address: busato@uniparthenope.it .

Giuli Francesco, Department of Economics, Roma Tre University, Via S. D'amico 77 - 00145 Rome, Italy. E-mail address: francesco.giuli@uniroma3.it.

Marchetti Enrico, University of Naples «Parthenope», Department of Economic and Legal Studies, Via G. Parisi 13, 80132 Naples, Italy. E-mail address: marchetti@uniparthenope.it.
\end{abstract}



a microfounded equilibrium perspective

ists a unique equilibrium with a tax evasion, consistent with the empirical estimates for the United States economy; ii) the model predicts a positive relationship between tax rate and evasion rate, while offering a solution to the so called Yitzhaki puzzle; iii) the «framing effect» plays a significant role in supporting these results; this is a distinctive characterstic of this class of model, typically not present in simple individual choice models. Furthermore, the model also allows us to investigate some potentially relevant effects of labor supply behavior on the tax compliance decisions.

\section{Key words:}

Tax evasion, fiscal policy, prospect theory.

JEL: E21; D03; D81; H03.

\section{Introduction}

Tax evasion, i.e., the illegal concealment of taxable activity, is a relevant issue in public economics, whose impact extends beyond developing economies, also interesting many developed economies, as testified by the most recent (although still tentative) estimations of «shadow» or undeclared economy around the world (see, e.g., Medina and Schneider 2018). Beyond the obvious difficulties related to the measurement of the phenomenon, tax evasion presents itself also as a complex theoretical issue, due to the interplay of many institutional, cultural and directly economic motivations behind the individual decisions to hide taxable resources.

Nevertheless, the elaboration of a good theory of tax compliance decisions is essential for designing a tax structure capable of deterring evasion (Hashimzade, 2013), and the economic analysis of this topic dates back to the pioneering work of Allingham and Sandmo (1972), followed by a conspicuous number of contributions of theoretical and empirical nature. The inclusion of tax evasion in standard models of individual choice (of microeconomic nature) brought about a number of interesting «puzzles», or unexpected or surprising results, that cast doubts on the ability of standard microeconomic theory (of individual choice) to properly explain the phenomenon. Prominent among these per- 
plexing results, there is the so-called Yitzhaki paradox or Yitzhaki puzzle (from Yitzhaki 1974): expected utility models (ET) endowed with a standard description of preferences' parameter tend to predict a decrease in the amount of tax evasion when the tax rate increases.

The subsequent literature followed different routes in order to overcome this (and others) problem. Beyond focusing on "context» or cultural elements, such as the tax morale, ${ }^{1}$ an important and a recent strand of research took advantage of the progresses of behavioral economics in trying to extend and enhance more traditional models of individual choice. ${ }^{2}$ Of particular interest is the attempt to integrate in the analysis of tax evasion probably the most successful and widely known behavioral theory of choice under risk, i.e. the Prospect Theory (PT) originally developed by Tversky and Kahneman (1992).

PT, in its more complete version, cumulative Prospect Theory, can be summarized by the following five assumptions: i) reference dependence: the carriers of utility are wealth gains and losses relative to some reference point; ii) declining sensitivity: the utility function is concave in the domain of gains and convex in the domain of losses; iii) loss aversion: losses are more salient than gains; iv) non-linear weighting of probabilities: agents facing uncertain situations overweight small probabilities but underweight large ones; v) susceptibility to framing effects: agents' preferences are influenced by the way a problem is presented. ${ }^{3}$ Our study aims at contributing to this line of research by integrating PT into a scheme which goes beyond the individual-choice framework mainly followed by the literature so far, with the main goal of providing an intuitive solution to the Yitzhaki puzzle.

We incorporate the main elements of PT into a representative-agent model of market (static) equilibrium. The central idea is to adopt an equilibrium approach in the spirit of Ciccarone and Marchetti $(2013)^{4}$ and to nest in the selected distinctive elements of tax compliance decisions described in al-Nowaihi and Dhami (2007). This scheme allows us to devise an analytical framework in which wider equilibrium interactions can be taken into account, in the light of a more structured micro-foundation analysis of the tax evasion phenomenon. In particular, we can include into the analysis decisions related not only to consumption but also to labor supply, so as to investigate the role of tax evasion along these dimensions and the ability of this extended framework to provide a solution of the Yitzhaki puzzle.

\footnotetext{
${ }^{1}$ See Torgler (2007) for an overview.

2 See, e.g., Yaniv (1999); Bernasconi and Zanardi (2004); Arcand and Rota Graziosi (2005); al-Nowaihi and Dhami (2007); Rablen (2010); Cullis et al. (2012). See also Pickhardt and Prinz (2013) or Hashimdaze at al. (2013) for a extensive surveys.

${ }^{3}$ For an exhaustive discussion see, e.g., Wakker (2010).

${ }^{4}$ Ciccarone and Marchetti (2013) elaborate an OLG model with signal extraction and a behavioral component in the agent's utility function. In our model we consider only a static economic environment.
} 
180 Maria Carmela Aprile, Francesco Busato, Francesco Giuli, Enrico Marchetti

Prospect Theory and tax compliance: a microfounded equilibrium perspective

The model's results present improvements on different topics and issues highlighted by the literature. The paper shows that in equilibrium the representative agent always chooses - under suitable conditions - a positive but limited amount of tax evasion. ${ }^{5}$ More specifically, the model possesses interior (closed form stationary) solutions under an exogenous and constant probability of being detected evading; this improves upon the assumption of endogenous probability, which is typically considered a weakness of individual choice models. Furthermore, the model can overcome the Yitzhaki puzzle in quantitative macroeconomic set-up. We develop a calibration exercise so as to properly fit the United States economy key characteristics, relevant for our issues, with particular attention to the evasion rate. Under this calibration, the model predicts that an increase in the tax rate leads to an increase in tax evasion, in line with the empirical evidence, while allowing us to investigate the relevance and the impact of the framing effect in the context of the tax compliance decision. We uncover an inverse relationship between the parameter determining the framing effect and that representing the weight of social customs and stigma. In other words, there exists of trade-off between these two dimensions in the context of tax evasion: a given level of tax evasion can be related either to a low level of framing coupled to a high level of social stigma or to a weak framing-high stigma combination. Furthermore, the slope of this trade off is affected by the elasticity of labor supply: the higher the elasticity, the lower the level of framing needed to support a given amount of tax evasion.

The paper is structured along the following lines. Section 2 describes the model. Section 3 develops the numerical exercise for the United States economy and discusses the results. Finally Section 4 offers some conclusion.

\section{The model}

The economy is populated by a representative agent and an exogenous fiscal authority which collects taxes on produced income, and tackles possible tax evasion schemes by enforcing deterrence policies and random audits; it furthermore balance its budget via wasteful public expenditure.

The representative agent has the utility function:

$$
u=\frac{c^{\gamma}}{\gamma}-\xi \frac{L^{\psi}}{\psi}+\beta v\left(W_{b, n D}\right)
$$

\footnotetext{
${ }^{5}$ The original al-Nowaihi and Dhami (2007) model derives a bang-bang solution for tax evasion; hence it properly describes and explains the phenomen at a microeconomic level, but is not well equipped for macroeconomic analysis.
} 
Where $C$ is real consumption of the unique type of good, $L$ is the amount of labor needed to produce it and $0<y<1, \psi>1, \xi>0$ and $\beta>0$ are parameters; the function $v($.$) , which includes the behavioral elements, is detailed in the$ sequel. The two quantities $C^{\gamma / \gamma}$ and $L^{\psi} / \psi$ are the traditional ones (utility form consumption stream and disutility for labor services). Each agent can be thought as consumer-producer, so that he/she carries out current production $Y$ by using the technology:

$$
Y=L
$$

The agent's disposable income is, in expected terms, denoted by $Y^{E}$, which takes into account taxation and an audit scheme.

The fiscal mechanism acts in the following way: gross resources $Y$ are taxable and the agent declares to the Internal Revenue Service (IRS) an amount $0<D<Y$. The government levies a tax on declared resources $D$ at the constant marginal rate $0<\tau<1$. When a taxpayer evades $0 \leq D_{t}<Y_{t}$, he/she is detected with probability $p \in(0 ; 1)$. If caught evading, the agent pays a constant surcharge $s>0$ on the outstanding tax liabilities $Y-D$. Thus his/her final amount of resources is given by the following binary scheme, $\bar{Y}=\left[Y^{N-D e t} ; Y^{N-N o m D}\right]$, where:

$$
\begin{array}{ll}
\text { Detected }(\sim p): & Y^{N-D s t}=(1-\tau) Y-s \tau(Y-D) \\
\text { Not Detected }(\sim 1-p): & Y^{N-N o m D}=Y-\tau D
\end{array}
$$

Then, the expected amount of net income stems from the linear projection:

$$
Y^{\bar{E}}=[1-p \tau(1+s)] Y-\tau[1-p(1+s)] D
$$

We now define the budget constraint:

$$
C \leq Y^{E}=[1-p \tau(1+s)] Y-\tau[1-p(1+s)]
$$

We can describe the features of the utility function (1) by following the general idea of Barberis and Huang (2009), i.e., that agent are usually subject to narrow framing, which is a well-known result of PT and of behavioural economics in general. In the EUT model, the agent evaluates a new gamble he/she is offered by merging the new gamble with other risks he/she is already facing, so as to determine its overall effect on the distribution of his/her future wealth or consumption. Then he/she evaluates the new distribution so as to ascertain possible improvements or worsenings.

Nevertheless, a wide experimental literature highlights many deviations from this behaviour: agents tend to evaluate a new gamble in isolation, separately from their other risks. This behavior is named "narrow framing" (Khanemann and Lovallo 1993; Khanemann 2003) because the decision taken by the agent can be affected by the way the decision problem is posited (framing effect). Also the new gamble is evaluated by considering the distribution of (the gam- 

a microfounded equilibrium perspective

ble's) outcomes taken alone, rather than considering the overall distribution of wealth/resources. In this sense (and coherently with Barberis and Huang 2009) we may assume that the agent derives utility from the gamble's contributions to the overall profile of wealth/resources and consumption (the term $\frac{d r}{\gamma}$, as in the standard models) and also directly from a narrow framing effect, as represented by the term $\beta v\left(w_{B, n D}\right)^{6}$ The parameter $\beta$ can then be interpreted as a quantitative measure of the narrow framing effect.

The term $v\left(w_{b n D}\right)$ (the value function, in $\mathrm{PT}$ terminology) in equation (1) is adapted from al-Nowaihi and Dhami (2007) and encapsulates the remaining four fundamental elements of (cumulative) PT: reference dependence, constant sensitivity, loss aversion, and probability weighting. ${ }^{7}$ We define the gains and losses as $W_{D, m D}=(\bar{Y}-R)$, where $R$ is the reference value of total resources/wealth and it is equal to the legal after-tax wealth:

$$
R=(1-\tau) Y
$$

Following al-Nowaihi and Dhami (2007), we define the gains-losses $W_{B M D}$ by including a social stigma component, $s_{w w}$, when the agent is caught evading. Hence $w_{D, n}$ can be split into:

$$
\begin{gathered}
W_{d e t}=-\left(s_{s o c} s\right)(Y-D) \\
W_{N-d e t}=\tau(Y-D)
\end{gathered}
$$

Our definition of $W_{d s t}$ is different from that adopted by al-Nowaihi and Dhami (2007): in their paper the expression for $w_{d s t}$ is: $\left(s_{s v c}+s \tau\right)(y-D)$, i.e., the social stigma is added to the tax surcharge. Nevertheless we justify our choice of $W_{d e t}$ by noting that, on one side, in the calibration analysis of section 3 $s_{v o c}$ plays the role of a deep parameter, which is not pinned down by independent empirical evidence. ${ }^{8}$ On the other hand, we also performed simulations with a version of the model where $W_{d s r}=-\left(s_{s u c}+s \tau\right)(Y-D)$ and the results do not qualitatively change.

\footnotetext{
${ }^{6}$ Notice that a rational agent - in the traditional sense of economic rationality - should only consider the term $\frac{c^{\gamma}}{y}$.

${ }^{7}$ Reference dependece implies that the agent is interested only in gains and losses form a reference point. Declining sensitivity makes the utility function concave in the domain of gains and convex in the domain of losses. Loss aversion captures the idea that, in the agent's eyes, losses loom larger that gains. Finally, non-linear probability weighting implies that the agent sistematically overestimates the probability of rare events, while underestimating the probability of more frequent ones.

${ }^{8}$ Furthermore, reliable empirical estimates of this parameter are difficult to obtain.
} 
As for the function $v$, it is defined as:

$$
\begin{aligned}
& v\left(W_{B_{M D}}\right)=E_{w}\left(W_{B, D}\right) \\
& =E_{w}\left(\begin{array}{rl}
W_{W-\text { Det }} \text { with } W_{N-\text { det }} & >0 \text { with probaility } 1-p \\
-\theta\left(-W_{\text {Det }}\right) \text { with } W_{D e t} & <0 \text { with probability } p
\end{array}\right.
\end{aligned}
$$

where the $E_{w}$ is an operator which combines the two branches $W_{N \text {-Dst }}$ and $W_{\text {set }}$ by means of the probability weighting function $w($.$) , whose argument is the true$ (objectively known) value of the probabilities $p$ and $1-p$. Our function $v$ is linear in its arguments $W_{B, m D}$ implying a constant sensitivity property as in Barberis et al. (2001). The parameter $\theta>1$ represents the loss aversion: possible losses $\left(w_{D, n D}<0\right)$ are more salient than equivalent gains $\left(w_{B n D}>0\right)$. The probability weighting function $w$ is chosen so as to meet the fourth requirement: non-linear weighting. The most common choice for the function $w$ is the one proposed by Prelec (1998):

$$
w(p)=e^{-\left[-\ln p y^{2}\right.} \text { for } p \in(0 ; 1] \quad w(0)=0 \text { and } \quad 0<b<1
$$
lows:

Given these specifications, we can compute the value function $v$ as fol-

$$
\begin{aligned}
& v\left(W_{b, n D}\right)=\left[w(1-p) \tau-w(p) \theta\left(s_{s o c} s \tau\right)\right](Y-D) \\
& =\left[e^{-[-\ln (1-p)]^{b}} \tau-e^{-\left(-\ln p_{3} b\right.} \theta\left(s_{w} s \tau\right)\right](Y-D) \\
& =h(Y-D)
\end{aligned}
$$

Where $h$ is a function of parameters only, and where the social stigma is represented by parameter $s_{s o c} \in(1 ; \infty)$.

\subsection{Model's equilibrium}

We characterize the model's equilibrium by solving the following problem:

$$
\begin{aligned}
& \max _{Y, D} U=\frac{[1-g \tau[1+s] Y-\tau[1-p(1+\Sigma) D]}{\gamma}-\xi \frac{z^{\psi}}{\psi}+\beta v(Y-D) \\
& \text { s. t. } D-Y \leq 0 ; \quad D \geq 0 ; \quad Y \geq 0
\end{aligned}
$$

We included in the utility function $U$ consumption $C$ as expressed by the agent's budget constraint (3) with equality. As for the three other constraints in (6), they allow us to investigate the possibility of interesting corner solutions, i.e. when it is $(Y>0 ; D=0)$ or when it is $Y=D>0$. Before moving to the discussion 

a microfounded equilibrium perspective

of the solution, Lemma 1 deals with the concavity of the utility function $U$ in problem (6), which is a delicate issue for this class of models.

Lemma 1: The function $U(Y, D)$ in problem (6) is strictly concave for $Y>0$ and $C>0$.

Proof: See Appendix.

The linearity of the constraint $D-Y \leq 0$ allows us to carry out the analysis of problem (6) in a standard fashion. To ensure economically reasonable solutions, we restrict ourselves to the case:

$$
h<0 \text { and } p(1+s)<1
$$

When $h<0$ the agent found him/herself in the region of losses (unless it is $Y=D)$ and $p(1+s)<1$ is a common assumption in this type of studies, given the small values of the detection probability commonly found in empirical analyses. ${ }^{9}$

Solutions of problem (6) are synthesized in:

Proposition 1: Optimal values of $Y$ and $D$ are related to the framing parameter $\beta$ and to the threshold values:

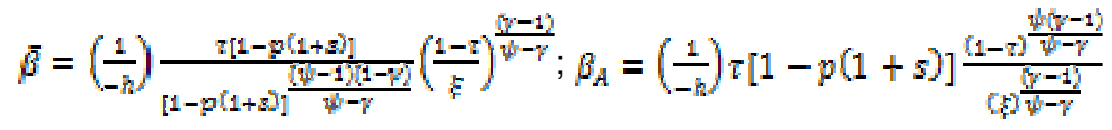

in the following way:

1) when: $\beta \leq \bar{\beta}$ the chosen values are: $D=0$ and

$$
Y \geq\left(\frac{(1-\tau)}{[1-p(1+v)]^{1-\gamma}}\right)^{\frac{1}{\psi-\gamma}}>0
$$

2) when: $\beta \in\left(\bar{\beta} ; \beta_{A}\right)$ the chosen values are:

$$
\begin{aligned}
& Y=\left(-(1-\tau) A_{0}\right)^{\frac{1}{\psi-1}} \\
& D=A_{1} Y-\frac{\left(-A_{2}\right)^{\frac{1}{\gamma-1}}}{\tau[1-p(1+z)]^{1-\gamma}}
\end{aligned}
$$

Where: $A_{0}=\frac{\beta h}{p r[1-p(1+s)]^{*}} ; \quad A_{1}=\frac{1-p r(1+s)]}{\tau[1-p(1+3)]}$

1) when $\beta \geq \beta_{A}>\bar{\beta}$ the chosen values are: $Y=D=\left(\frac{(1-\tau)}{k}\right)^{\frac{1}{\psi-\gamma}}>0$.

Proof: See Appendix.

\footnotetext{
${ }^{9}$ This is also coherent with the numerical analyses carried out in the subsequent sections.
} 

tion is:

It is worth noticing that, when the agent is fully rational, i.e. $\beta=0$, the solu-

$$
\left(\frac{\left[1-p c(1+a] \eta^{\gamma}\right.}{k}\right)^{\frac{1}{p-\gamma}}=Y_{\beta=0}>0
$$

and the evasion is full: $D=0$, as it is certainly $\beta=0<\bar{\beta}$.

In the market equilibrium we impose a government balanced budget, i.e., $G=\tau[1-p(1+s)] D+p \tau(1+s) Y$. This implies that:

$$
L=Y=C+G
$$

\section{Calibration and model analysis}

We apply the model to the United States (US) economy, as plenty of empirical estimates of the relevant parameters are available for this economic system. The aim of our analysis is threefold:

- we first want to verify that our model, given the average tax rate $z$ for the US, can replicate the empirically estimated amount of tax evasion (as represented by the ratio $\frac{Y-D}{Y}$ ) for plausible values of the remaining parameters.

- Next, we perform a numerical exercise in order to determine the response of our model to changes in tax rate $\tau$, i.e. to test its ability to solve the Yitzhaki puzzle. We explore this issue under two different assumptions on the features of labour supply: an economy with a rigid labor supply and another one in which an elastic labor supply prevails. $^{10}$

- We also explore the model's behaviour when the two most important parameters (for our approach, i.e.: $\beta$ and $s_{s o c}$ ) changes. We then consider different values for $\beta$ and $s_{w}$ and verify the existence of an positive relationship between $\frac{Y-D}{Y}$ and $\tau$.

There are nine parameters in the model: $\gamma, \xi, \psi, p, s, b, \theta, s_{v a c}$ and $\beta$. We first assume a log specification for consumption utility (i.e. $\gamma=0$ ), which is commonly adopted in standard macroeconomic modelling (our results would not qualita-

\footnotetext{
${ }^{10} \mathrm{~A}$ rigid labor market is characterised by a high $\psi$, while an elastic one by a low $\psi$.
} 

a microfounded equilibrium perspective

tively change under a strictly positive value of $\gamma$ ). Equilibrium conditions now read:

$$
\begin{gathered}
Y=\left(-(1-\tau) A_{0}\right)^{\frac{1}{\psi-1}} \\
D=A_{1} Y+\frac{p}{\beta h}
\end{gathered}
$$

$$
\text { Where: } A_{0}=\frac{\beta h}{p[1-p(1+2)]}: \quad A_{1}=\frac{1-p r t(1+3)]}{\eta[1-p(1+B)]}
$$

In order to undertake a rigorous calibration exercise, we pin down five parameters from existing literature $(\psi, p, s, b, \theta)$, relying on commonly accepted figures. The remaining two parameters are the social stigma $s_{s o c}$ and the framing parameter $\beta$; these are truly deep parameters and we work on these, to understand whether reasonable figures are capable to endorse our targets.

It is first necessary to find a figure for the evasion rate $\frac{Y-Z}{Y}$. This is a challenging empirical estimate due to the natural difficulty of estimating an unofficially reported quantity. Here we use as a benchmark the widely accepted estimates of the ratio of the underground economy over total GDP (for the US), provided by Schneider and Enste (2000) and Schneider et al. (2010), who set this ratio in the range of $8.4 \%$ to $8.8 \%$ in the recent years. This should be properly thought of as the ratio of the aggregate income evaded over total income. In particular, taking into account the possibility of specific forms of evasion for wealth (such as migration in fiscal paradise, etc.) it appears reasonable to assume $8.8 \%$ as a plausible figure.

As for the benchmark value of the tax rate $\tau$, we first rely on official data from the Bureau of Economic Analysis ${ }^{11}$ to compute the stationary Government Receipt/GDP ratio: GovE/Y $=0.2755$, over the period 1950-2011 and then set $\tau=G o v E / Y$.

We discuss, next, the calibration of the other five parameters.

1. The loss aversion parameter $\theta$ is set to $\theta=2.25$, as justified by a wide amount of empirical research (e.g. Tversky and Kahneman, 1992).

2. The parameter of the Prelec weighting function is set to $b=0.35$, which is the benchmark value in al-Nowaihi and Dhami (2007) analysis.

3. The surcharge rate or penalty rate $s$ is set to $s=0.5$, which is a viable empirical estimate for the additional payment after an audit in the USA (see Alm et al. 1992; Andreoni et al. 1998).

\footnotetext{
${ }^{11}$ The tables are: GDP (nominal), NIPA table 1.1.5; Government receipt, (nominal) NIPA table 3.1. GovE and $Y$ are computed as long run averages.
} 
4. Probability of being detected $p$ is set to $p=0.02$, which is the average value of the range of realistic values for the US economy: $p \in[0.01 ; 0.03]$, as reported by al-Nowaihi and Dhami (2007), ${ }^{12}$

5. Parameter $\psi$ is a measure of inverse total labor supply elasticity, which we use for discussing two scenarios for the labor market. A rigid labor market scenario (i.e. $\psi=7$ or 10 ) and an elastic labor market scenario (i.e. $\psi=1,2$ ). The latter seems consistent with the estimates of Kimball and Shapiro (2008) who obtain a Frisch elasticity close to 1 .

Finally, the scaling parameter $\xi$ of the disutiltiy of labor is calibrated so as to ensure that, in the subsequent analysis all endogenous variables (notably $Y$ and $D$ and their ratios) are positive and, when required, capable to match the targets.

We can now use the two parameters controlling the social stigma and the framing effect $s_{s w c}$ and $\beta$, respectively. The calibrations for the two labor market scenario are summarized in the following table and discussed in the next section.

Table 1

Scenario \#1: Rigid labor market $. \psi=7: 10$

\begin{tabular}{|c|c|c|c|}
\hline Fixed parameters & $\tau=0.2755$ & $s=0.5$ & $\theta=2.25$ \\
\hline & $p=0.02$ & $b=0.35$ & \\
\hline Adjusted parameters & $\overline{s_{s u c}: \beta ; \xi}$ & & \\
\hline \multicolumn{4}{|c|}{ Scenario \#2: Elastci labor market $. \psi \psi=1.2$} \\
\hline \multirow[t]{2}{*}{ Fixed parameters } & $\tau=0.2755$ & $s=0.5$ & $\theta=2.25$ \\
\hline & $p=0.02$ & $b=0.35$ & \\
\hline Adjusted parameters & $s_{s u c}: \beta ; \xi$ & & \\
\hline
\end{tabular}

In this framework we investigate the model's response (with attention to the equilibrium evasion rate) after changes in the tax structure; we also analyze the role played by the agents' main behavioral components ${ }^{s}$ sus and $\beta$. From a technical perspective, we undertake a perturbation of the parameter space in the two scenarios.

\footnotetext{
${ }^{12}$ See also Alm et al (1992); Andreoni et al. (1998) and Bernasconi (1998).
} 


\subsection{Scenario \# 1: PT in a rigid labor market}

We consider, in this context, two different calibration schemes, differing only along the social stigma and framing dimensions: the first one refers to an economy with relatively low social stigma in evasion and relatively strong framing effects; the second one represents an economy with relative high social stigma in evasion and relatively low framing effects. Table 2 summarizes the first calibration scheme:

Table 2

\begin{tabular}{|l||l||l||}
\hline \multicolumn{2}{|l||}{ Strong framing effect; low social stigma } & \\
\hline \hline Standard calibration: & as in Table 1 & $\psi=7$ \\
\hline \hline Labor market & $\xi=0.04$ & $\beta=6.88$ \\
\hline \hline Deep parameters & $s_{\text {soc }}=4$ & $\beta=4$ \\
\hline
\end{tabular}

By plugging this calibration into equations (9)-(10) we confirm that we are reasoning around the empirically estimated evasion rate $\frac{Y-D}{Y} \cong 0.088$. A simulation of the numerical versions of (9)-(10) is presented in Figure 1 below.

Panel A shows the positive relation between tax rate increases and evasion rate, which is our proposed solution to the Yitzhaki puzzle, qualitatively in line with the empirical evidence. In addition, once the tax rate and the evasion rate are pinned down, it is possible to draw an inverse relation between $\beta$ and $s_{\text {swe }}$ (Panel B).

It interesting to mention that qualitatively similar results arise in a calibration with weak framing effects and high social stigma, such as the one shown in Table 3 below. The model predicts the empirically consistent evasion rate, and solves the Yitzhaki puzzle (positive relationship between $\frac{Y-Z}{Y}$ and $\tau$ ). 


\section{Figure 1}

Panel A: the relationship between $D / Y$ and $(Y-D) / Y$ as dependent variables and $\tau$ where the values of $\left(\beta, \xi, \psi, p, s, b, \theta, s_{s a c}\right)$ are those of Table 2, with $\tau \in[0,25,0.5]$. Panel B: the trade-off between $\beta$ and $s_{s w}$ according to equation (11) and the values of $\left(\frac{y-E}{y}, \xi, \psi, \tau, p, s, b, \theta\right)$ of Table 2. Notice that in both Panels the value of $h$ is always negative.
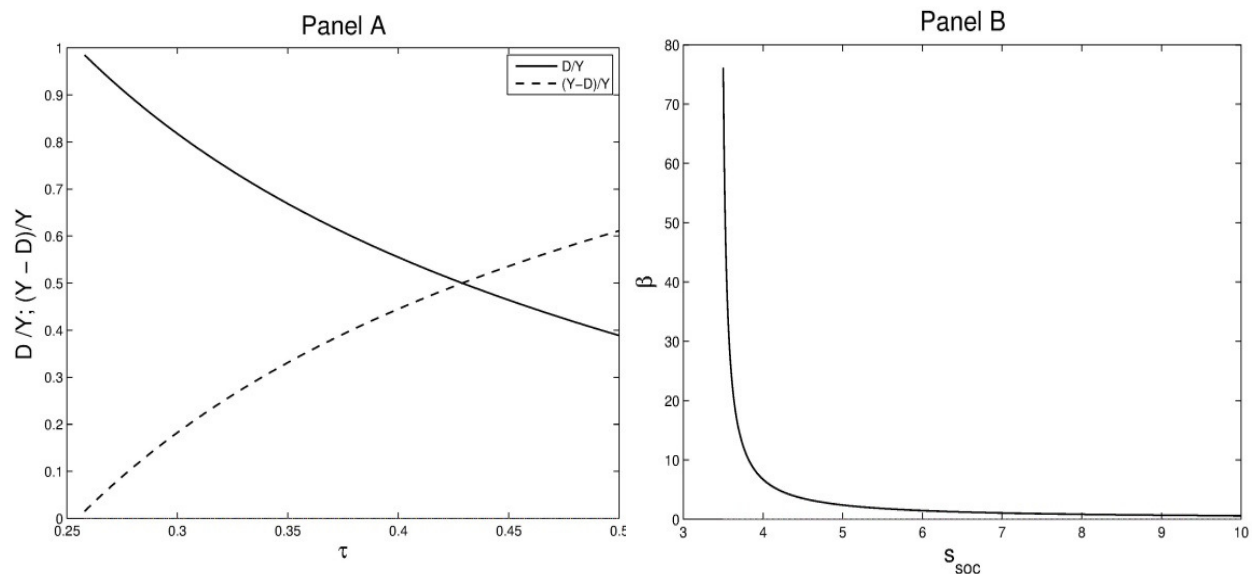

Table 3

\begin{tabular}{|l||l|l||}
\hline Weak framing effect; high social stigma \\
\hline Standard calibration: & as in Table 1 & \\
\hline \hline Labor market & $\xi=0.03$ & $\approx=10$ \\
\hline \hline Deep parameters & $s_{s, c}=\mathbf{8}$ & $\beta=0.8975$ \\
\hline
\end{tabular}

The results we just presented suggest that there exist a trade-off between $s_{w v e}$ (included in $h\left(s_{w v}\right)$ ) and $\beta$. This comment can be formally derived from the stationary equations for $Y$ and $D$; computing $\frac{Y-D}{Y}$, after some algebra, we obtain the following relation:

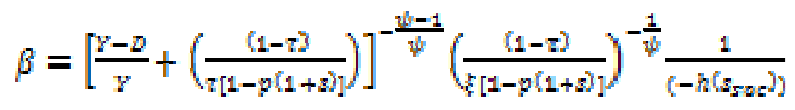



a microfounded equilibrium perspective

This means that the Yitzhaki puzzle (i.e., the slope of the relationship between $((Y-D) / Y$ and $T$ ) can be solved by analyzing the behavior of equation (11). In other words in our macroeconomic model, evasion rates positively respond to an increase in tax rates (as evidences suggest) either with strong framing effects and low social stigma, or with weak framing effects and high social stigma. We think, however, it would be interesting to explore the possibility to preserve this result with an even smaller framing effect.

\subsection{Scenario \# 2: PT in an elastic labor market}

The analysis of the elastic labor market scenario can be carried out along the same lines of the previous section. Table 4 presents the calibration for an elastic labor market (i.e. a low value of $\psi$, coherent with macroeconomic estimates):

Table 4

\begin{tabular}{|l||l|l|}
\hline \multicolumn{2}{|l|}{ very weak framing effect; high social stigma } \\
\hline \hline Standard calibration: & as in Table 1 & \\
\hline \hline Labor market & $\xi=0.03$ & $\psi=1.2$ \\
\hline \hline Deep parameters & $s_{\text {sw }}=9$ & $\beta=0.057555$ \\
\hline
\end{tabular}

The numerical results are presented in Figure 2:

Panel A shows a positive relationship between $\tau$ and $\frac{Y-D}{Y}$, analogous to that of Figure 1; the trade-off between $\beta$ and $s_{s w c}$ is also confirmed (Panel B).

There are two interesting results. First, the model offers an empirically consistent prediction for the $\left(\tau ; \frac{Y-Z}{y}\right)$ relationship for a smaller range of $\tau$ rates. Second, in a "high-elasticity economy» a very small deviation from rationality is sufficient for having a positively sloped relation between tax rates and evasion rates; we think that this a particularly welcome result. 
Figure 2

Panel A: the relationship between $D / Y$ and $(Y-D) / Y$ as dependent variables and ${ }^{2}$ where the values of $\left(\beta, \xi, \psi, p, s, b, \theta, s_{w w}\right)$ are those of Table 4, with $\tau \in[0.275,0.313]$. Panel B: the trade-off between $\beta$ and $s_{s o v}$ according to equation (11) and the values of $\left(\frac{Y-D}{Y}, \xi, \psi, \tau, p, s, b, \theta\right)$ of Table 2. Notice that in both Panels the value of $h$ is always negative
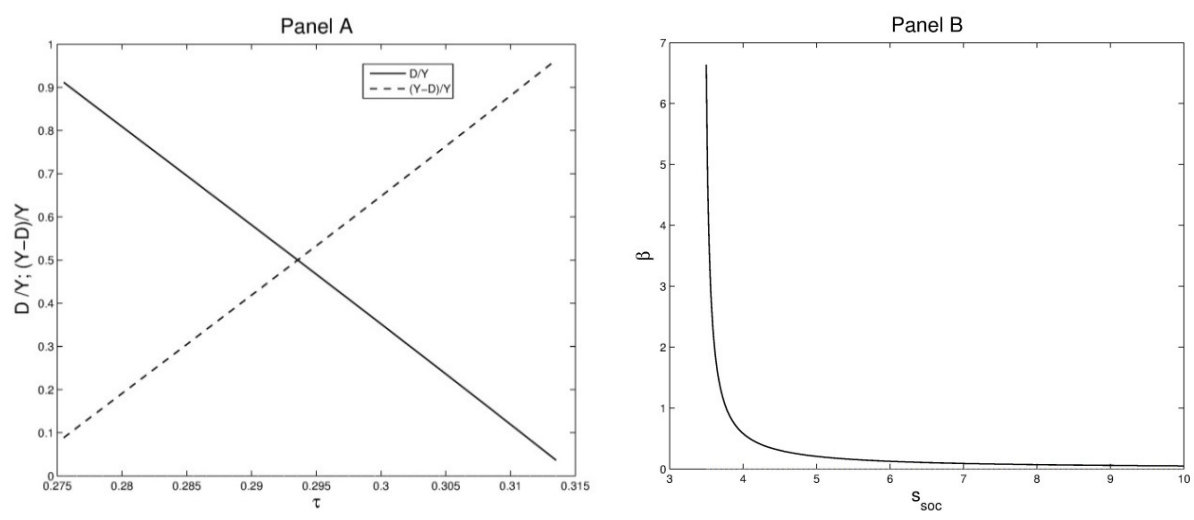

\subsection{Discussion: the economic intuition}

We think that this model makes an interesting point in theory, with a straightforward economic intuition. It is convenient to discuss the mechanism by recalling the equilibrium values of $D$ and $Y$, as given by equations (9)-(10) and taking the ratio $\frac{D}{Y}$ :

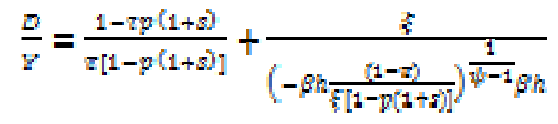

Since $h<0$, we rewrite the ratio as:

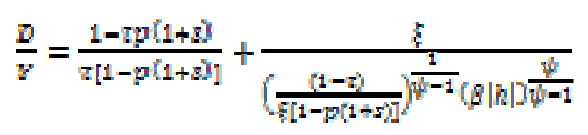

This last expression allows us to discuss the economic mechanism. Consider, first, a model without $\mathrm{PT}$, i.e. $\beta=0$, and notice that in this case $\frac{D}{Y} \rightarrow-\infty$; 

a microfounded equilibrium perspective

this means that $D=0$, in the sense that the tax payer will declare nothing. This is the corner solution that depicts the puzzling question «why do people pay taxes?».

When PT is included (i.e. imagine we make $\beta$ slowly increasing), the loss aversion component incentivates the tax payer to declare more. This is a key result of our model, which improves upon the current state of the art. While in alNowaihi and Dhami (2007), under constant detection probability, the agent switches from one corner to the other (no compliance, full compliance), our framing effect (in the spirit of Barberis and Huang 2009) convexifies, through the market mechanism, the equilibrium compliance choice. In our model, the additive structure of the utility function (1) allows the agents to compare the contributions of the choice variables (e.g., $D$ and $Y$ ) to the different additive components, so as to prevent the bang-bang solution. But the introduction of the additive elements into (1) requires, in its turn, to cast the analysis in a proper context of market equilibrium. More precisely, the reason why in our model evasion increases as tax rate raises stems from the fact that the tax payer, when young, feels poorer: then he/she will produce less and send a smaller amount of resources for consumption in the next period. The agent also foresees a lower level of consumption utility and finds himself/herself in the region of losses $(h<0)$; hence he/she is keen in taking more risk and therefore evades more.

In a flexible economy (i.e. the elastic labor market, scenario \#2), tax payers react more quickly to changes in fundamentals. Consider an increase in $\tau$. First, the young are willing to work less (and therefore to produce less); as a direct consequence, they will declare less income. Since the equilibrium decisions are strongly sensitive and reactive, it is sufficient a relatively small framing effect (i.e. $\beta$ ) to activate the mechanism previously discussed. This effect is also evident by direct inspection of equations (9), (10) and (12): a low $\psi$ implies a high value of the exponent in the terms of these equations and this, for example in the case of $Y$ in (9), means that a change in $\tau$ brings about a stronger response of the equilibrium output.

Finally, the origin of the trade-off between $\beta$ and $s_{s w}$ can also be explained by a straightforward argument. For having an economically viable solution, the term $h$ in the equilibrium equations (9)-(10) must be negative; as the same term is equal to: $h=e^{-\left(-\ln [1-p y)^{b}\right.} \tau-e^{-(-\ln p\}^{b}} \theta\left(s_{s o c} s c\right)$ and it is multiplied by $\beta$, it is evident that, for matching the empirical target of $\frac{Y-D}{F}$, a reduction of $\beta$ can be compensated by an increase of $s_{s o c}$. 


\section{Conclusions}

This paper presents a novel modelling framework in which PT is integrated into an otherwise simple and standard model of market equilibrium with an aggregate-representative agent, in the context of tax compliance choices. The aim is to tackle the main issues and puzzles of the tax evasion/compliance behavior with a theoretical instrument which is innovative with respect to previous attempts on the same route. The market equilibrium environment allows us to define the choice problem so as to take into account all the relevant features of PT and in particular the framing effect, which proved to be difficult for the existing models based on the individual-choice approach.

In our model the role of the framing effect is encapsulated into a single parameter $(\beta)$, and this is in line with the general strategy recently proposed by Rabin (2013) in order to extend and increase the explanatory power of current economic theory. This strategy, called PEEM (portable extensions of existing models), is based on the modification of an existing model by means of different psychological assumptions to be represented in terms of parameter values. In our scheme, by setting the framing parameter $\beta$ to zero, a completely standard model would result, so that the comparison with the standard theory is made straightforward. By assuming a positive value for the parameter $\beta$, behavioral PT elements can play a role, and we can show that the resulting equilibrium can be coherent with quantitative estimations of the amount evaded in US economy. That is, the numerical version of the model efficiently predicts the overall amount of tax evasion and generates a positive relationship between the tax rate and the evasion rate, thus providing a solution for the Yitzhaki puzzle. Furthermore, we explore the interaction of the framing effects - as represented by $\beta$ - with the social stigma, and find that there exists a trade-off between the two phenomena: the same amount of tax evasion (relative to the GDP) is compatible either with a low level of framing coupled to a high level of social stigma or with a low framing - high stigma combination.

As the model includes the equilibrium choice of the labor input to be used in production, we can investigate the role of the elasticity of labor supply in shaping the tax compliance behavior. We find that in the presence of a highly elastic labor supply, even a very small level of framing, and hence a very small deviation from standard rational behavior, can support the observed level of tax evasion, provided that the social stigma is sufficiently strong. This is due to the fact that in a high-elasticity economy the agents reactions are stronger, and this magnifies the effects predicted by PT. 


\section{References}

1. Allingham, M.G., Sandmo, A. 1972. Income tax evasion: a theoretical analysis. Journal of Public Economics, 1, 238-323.

2. Alm, J., McClelland, G.H., Schulze, W.D. 1992. Why do people pay taxes? Journal of Public Economics, 48, 21-38.

3. Andreoni, J., Erard, B., Feinstein, J. 1998. Tax Compliance. Journal of Economic Literature, 36, 818-860.

4. Arcand, J., Rota Graziosi, G. 2005. Tax Compliance and Rank Dependent Expected Utility. The Geneva Risk and Insurance Review, 30, 57-69.

5. Barberis, N. Huang, M. 2009.Preferences with frames: A new utility specification that allows for the framing of risks. Journal of Economic Dynamics and Control, 33, 1555-1576.

6. Barberis, N. Huang, M., Santos, T. 2001. Prospect Theory and Asset Prices, Quarterly Journal of Economics, 116, 1-53.

7. Bénassy, J. P., 1999. Analytical solutions to a structural signal extraction model: Lucas 1972 revisited. Journal of Monetary Economics 44, 509-521.

8. Bernasconi, M. 1998. Tax evasion and orders of risk aversion. Journal of Public Economics, 67, 123-134.

9. Bernasconi, M., Zanardi, A. 2004. Tax evasion, tax rates and reference dependence. FinanzArchiv, 60, 422-445.

10. Ciccarone, G., Marchetti, E. 2013. Rational expectations and loss aversion: potential output and welfare implications. Journal of Economic Behavior and Organization, 86, 24-36.

11. Cullis, J., Jones, P., Savoia, A., 2012. Social norms and tax compliance: Framing the decision to pay tax. The Journal of Socio-Economics, 41, 159168.

12. Dhami, S., al-Nowaihi A. 2007. Why do people pay taxes? Prospect theory versus expected utility theory. Journal of Economic Behavior \& Organization, 64, 171-192.

13. Hashimdaze, N., Myles, G., Tran-Nam, B. 2013. Applications of behavioural economics to tax evasion. Journal of Economic Surveys, 27, 941-977.

14. Kahneman, D. 2003. Maps of Bounded Rationality: Psychology for Behavioral Economics, American Economic Review, 93, 1449-75.

15. Kahneman, D., Lovallo, D. 1993. Timid Choices and Bold Forecasts: A Cognitive Perspective on Risk Taking. Management Science, 39, 17-31. 
16. Kimball, M., Shapiro, M. 2008. Labor Supply: Are the Income and Substitution Effects Both Large or Small? NBER Working Paper No. 14208.

17. Lucas, R., 1972. Expectations and the neutrality of money. Journal of Economic Theory 4, 103-124.

18. Prelec, D. 1998. The probability weighting function, Econometrica, 60, 497528.

19. Rabin, M. 2013. An approach to incorporating psychology into economics. American Economic Review, 103, 617-22.

20. Rablen, M.D. 2010. A reference-dependent model of tax evasion and exchange equity. Public Finance Review, 38, 282-305.

21. Schneider, F., Enste, D. 2000. Shadow Economies: Size, Causes and Consequences. Journal of Economic Literature, 38, 77-114.

22. Schneider, F. Buhen, A. Montenegro, C. 2010. New estimates for the shadow economies all over the world, International. Economic Journal, 24, 443-461.

23. Torgler, B. 2007. Tax Compliance and Tax Morale: A Theoretical and Empirical Analysis. Edward Elgar, Cheltenham.

24. Tversky, A., Kahneman, D. 1992. Advances in Prospect Theory: Cumulative Representation of Uncertainty. Journal of Risk and Uncertainty, 5, 297-323.

25. Yaniv, G. 1999. Tax compliance and advance tax payments: a prospect theory analysis. National Tax Journal, 52, 753-764.

26. Yitzhaki, S. 1974. A note on income tax evasion: a theoretical analysis. Journal of Public Economics, 3, 201-202.

\section{Appendix}

Proof or Lemma 1: The Hessian matrix $H$ of $U(Y, D)$ in problem (6) is:

$$
\begin{gathered}
H=\left[\begin{array}{ll}
a_{11} & a_{12} \\
a_{21} & a_{22}
\end{array}\right] \\
a_{11}=(\gamma-1)[1-p \tau(1+s)]^{2} c^{\gamma-2}-(\psi-1) \xi Y^{\psi-2} \\
a_{12}=a_{21}=-(\gamma-1) \tau[1-p(1+s)][1-p \tau(1+s)] c^{\gamma-2} \\
a_{22}=(\gamma-1) \tau^{2}[1-p(1+s)]^{2} c^{\gamma-2}
\end{gathered}
$$




where from the budget constraint it is:
$C=[1-p \tau(1+s)] Y-\tau[1-p(1+s) D \geq 0$. For $U$ to be strictly concave, matrix $H$ must be negative definite, that is the following conditions must be satisfied:

$$
a_{11}<0: \quad \mid H \|>0
$$

As it is $y \in(0 ; 1)$ and $\psi>1$, the first condition is satisfied for strictly positive values of $Y$ or $C$. As for the second one, note that the determinant of the Hessian $H$ is:

$$
|H|=(1-\gamma)(\psi-1) \tau^{2}[1-p \tau(1+s)]^{2} \xi C \gamma-2 Y^{\psi-2}>0
$$

For $Y, C>0$.

Proof or Proposition 1: The Lagrangian function of problem (6) is:

$$
\mathcal{L}=\frac{[[1-p(1+s)] Y-\tau[1-p(1+s) D]]^{\gamma}}{\gamma}-\xi \frac{L^{\psi}}{\psi}+\beta v(Y-D)+\lambda(Y-D)
$$

and, given lemma 1, optimality conditions for the problem are:

$$
\begin{aligned}
& \frac{d E}{d Y} \leq 0 \\
& \frac{d L}{d D} \leq 0 \\
& \lambda(Y-D)=0 \\
& Y \frac{d E}{d Y}=0 \\
& D \frac{d E}{d D}=0
\end{aligned}
$$

We now consider different cases:

Case l) $D=0$ and $Y>0$ : positive production/income and full evasion. From (14) and (16)-(17) we obtain:

$$
\begin{gathered}
{[1-p r(1+s)]\left[[1-p \tau(1+s)] Y \gamma-1-\xi Y^{\psi-1}+\beta h=0\right.} \\
-\tau[1-p(1+s)][[1-p(1+s)] Y]^{\gamma-1} \leq \beta h
\end{gathered}
$$

Hence we can substitute the term $-\tau[1-p(1+s)][1-p \tau(1+s)] Y y^{-1}$ in (18) so that it is:

$$
(1-\tau)[1-p(1+s)][11-p \tau(1+s)] Y\}^{\gamma-1} \leq E Y^{\psi-1}
$$

As $Y>0$ and $Y^{\psi-\gamma}$ is monotonically increasing in $Y$, this inequality can be expressed in this way: 


$$
Y \geq\left(\frac{(1-\tau)}{\left.Y_{1}-p(1+s)^{1-\gamma}\right)^{\frac{1}{\psi}-\gamma}}=Y_{A}\right.
$$

We then insert the lower bound $Y_{A}$ into (19):

$$
-\tau[1-p(1+s)]\left\{\left(\frac{1-\tau}{\varepsilon}\right)^{\frac{1}{\psi-\gamma}}[1-p \tau(1+s)]^{\frac{\hat{\psi}-1}{\psi-\gamma}}\right\}^{\gamma-1} \leq \beta h
$$

and solve with respect to $\beta$ :

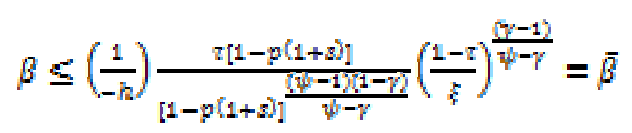

Case II) $Y, D>0$ and $D-Y<0$. In this case, from (15) it is $\lambda=0$ and from (16)-(17) we obtain:

$$
\begin{gathered}
{[1-p(1+s)]\left\{[1-p \tau(1+s)] Y-\tau[1-p(1+s)] D \gamma^{\gamma-1}-\xi Y^{\psi-1}=-\beta h\right.} \\
-\tau[1-p(1+s)]\{[1-p(1+s)] Y-\tau[1-p(1+s)] D\}^{\gamma-1}=\beta h
\end{gathered}
$$

This system of equations can be solved so as to obtain equations (7) and (8). The explicit form of the solution of $D$ is hence:

$$
D=\frac{1}{\tau[1-p[1+s]]}\left\{\left[\frac{(1-\tau)\left(-p_{n}\right)}{\tau[1-p(1+s)]}\right]^{\frac{1}{\psi-1}}[1-p \tau(1+s)]-\left[\frac{-\rho_{h}}{\tau[1-p(1+s)]}\right]^{\frac{1}{\gamma-1}}\right\}
$$

and given $D>0$ and $-\beta h>0$, it must be:

$$
\begin{gathered}
f_{1}(-\beta h)<f_{2}(-\beta h) \\
f_{1}=\left[\frac{(1-\tau)(-\beta h)}{\tau[1-p(1+2)]}\right]^{\frac{1}{\psi-1}}[1-p \tau(1+s)] \\
f_{2}=\left[\frac{-\beta h}{\tau[1-p[1+s)]}\right]^{\frac{1}{\gamma-1}}
\end{gathered}
$$

Both functions $f$ are positive and $f_{1}$ is monotonically increasing in $-\beta h$ while $f_{2}$ is monotonically decreasing in the same argument. They intersect at:

$$
-h \bar{\beta}=\tau[1-p(1+s)][1-p(1+s)]^{\frac{(j-1) / W-12}{\psi-\gamma}}\left(\frac{1-\tau}{t}\right)^{\frac{\mid \gamma-12}{\psi-\gamma}}
$$

Hence, for inequality (20) to hold, it must be:

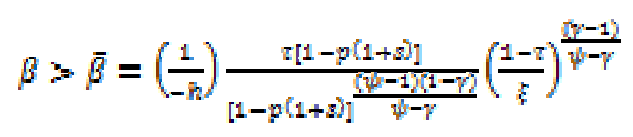


198 Maria Garmela Aprile, Francesco Busato, Francesco Giuli, Enrico Marchetti

Prospect Theory and tax compliance: a microfounded equilibrium perspective

Case III) $Y=D>0$, i.e. positive production and no tax evasion. From (15) it is $\lambda \geq 0$ and equations (16)-(17) are now:

$$
\begin{aligned}
& {[1-p \tau(1+s)][1-\tau] Y \gamma^{\gamma-1}-\xi Y^{\psi-1}+\beta h+\lambda=0} \\
& -\tau[1-p(1+s)][11-\tau] Y \gamma^{\gamma-1}-\beta h-\lambda=0
\end{aligned}
$$

The solution of income/production is hence:

$$
Y^{* 4}=\left(\frac{1-\tau}{\xi}\right)^{\frac{1}{y-\gamma}}>0
$$

which can be substituted in one of the (21)-(22) yielding:

$$
\lambda=-\tau[1-p(1+s)]\left\{(1-\tau)\left[\frac{(1-\tau) \gamma}{t}\right]^{\frac{1}{\psi}-\gamma}\right\}^{\gamma-1}-\beta h \geq 0
$$

The inequality can also be expressed in this way:

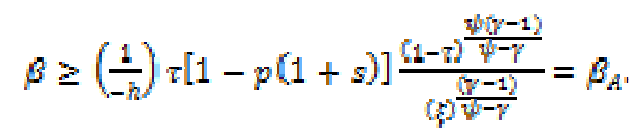

Straightforward computation shows that: $\beta_{A}>\bar{\beta}$, due to the assumption $p(1+s)<1$. 